\title{
Comparison of Three FE-FV Numerical Schemes for Single- and Two-Phase Flow Simulation of Fractured Porous Media
}

\author{
H. M. Nick - S. K. Matthäi
}

Received: 16 February 2010 / Accepted: 10 June 2011 / Published online: 30 June 2011

(C) The Author(s) 2011. This article is published with open access at Springerlink.com

\begin{abstract}
We benchmark a family of hybrid finite element-node-centered finite volume discretization methods (FEFV) for single- and two-phase flow/transport through porous media with discrete fracture representations. Special emphasis is placed on a new method we call DFEFVM in which the mesh is split along fracture-matrix interfaces so that discontinuities in concentration or saturation can evolve rather than being suppressed by nodal averaging of these variables. The main objective is to illustrate differences among three discretization schemes suitable for discrete fracture modeling: (a) FEFVM with volumetric finite elements for both fractures and porous rock matrix, (b) FEFVM with lower dimensional finite elements for fractures and volumetric finite elements for the matrix, and (c) DFEFVM with a mesh that is split along material discontinuities. Fracture discontinuities strongly influence single- and multi-phase fluid flow. Continuum methods, when used to model transport across such interfaces, smear out concentration/saturation. We show that the new DFEFVM addresses this problem producing significantly more accurate results. Sealed and open single fractures as well as a realistic fracture geometry are used to conduct tracer and water-flooding numerical experiments. The benchmarking results also reveal the limitations/mesh refinement requirements of FE node-centered FV hybrid methods. We show that the DFEFVM method produces more accurate results even for much coarser meshes.
\end{abstract}

\footnotetext{
H. M. Nick $(\varangle)$

Department of Earth Science and Engineering, Imperial College London,

South Kensington Campus, Exhibition Road, SW7 2AZ, London, UK

e-mail: nick@geo.uu.nl
}

\section{H. M. Nick}

Department of Earth Sciences-Geochemistry, Utrecht University, P.O. Box 80.021, 3508 TA,

Utrecht, The Netherlands

\section{S. K. Matthäi}

Department of Mineral Resources \& Petroleum Engineering,

Montan University of Leoben,

Max Tendler Str. 4, 8700 Leoben, Austria

e-mail: stephan.matthai@unileoben.ac.at 
Keywords DFM · FEM $\cdot$ FVM $\cdot$ Two phase flow $\cdot$ Discrete fracture and matrix model

\section{Introduction}

Accurate simulation of flow and transport through fractured rock requires a model which captures the physics of the flow processes, as well as an accurate representation of the flow geometry. Discrete fracture and matrix (DFM) models use the single-continuum approach in which both fractures and porous rock (i.e., matrix) have discrete representations in the model, and were developed for this purpose (Kim and Deo 2000; Karimi-Fard and Firoozabadi 2003; Dietrich et al. 2005; Matthäi et al. 2010; Geiger et al. 2009), as well as to perform upscaling studies (Gong et al. 2008; Matthäi and Nick 2009). In this approach, numerical modeling of fracture-matrix transfer processes needs special attention because the porous medium terminates abruptly at the fracture-matrix interface. Due to differences in the multiphase flow properties, saturation discontinuities may occur (van Duijn et al. 1995). The experiments of Su and Nimmo (2003) and Rangel-German and Kovscek (2003) show the influence of such material discontinuities and their barrier effect on two-phase flow. Imbibition experiments conducted by Rangel-German and Kovscek (2003) on a single fracture in contact with a low permeability rock, reveal the effect of capillary pressure and velocity magnitude on the imbibition process at the interface.

The effect of capillary pressure on two-phase flow DFM simulation has previously been addressed by Monteagudo and Firoozabadi (2004), Niessner et al. (2005), Mikyska (2005), and Reichenberger et al. (2006). In particular, Hoteit and Firoozabadi (2008a,b) applied a new formulation to capture transfer by combining the mixed finite element with the discontinuous Galerkin (DG) method. Hoteit and Firoozabadi (2008a,b) also use lower dimensional elements without the assumption of cross-flow equilibrium between fractures and neighboring matrix cells. Recently, Eikemo et al. (2009) proposed a DG method for advective transport in fractured porous media. They studied its accuracy and utility for different test cases, but observed that the accuracy decreases for the case of a high permeability contrast between the finite elements. This discussion highlights that this problem still has scope to explore alternative solutions for fracture-matrix transfer in search of physically realistic models.

In this study, we present a comparison between two FEFV methods and a new FEFVM with embedded discontinuity hybrid approach (DFEFVM) for purely advective flow in fractured porous media. The DFEFVM is introduced and verified by Nick and Matthäi (2011) for the simulation of single-phase flow and transport in inhomogeneous porous media. We achieve the embedded discontinuity approach by adding extra nodes/degree of freedom at the fracture-matrix interfaces and solve the equations accordingly. Our main goal is to contrast and compare of these methods. For this purpose, three numerical schemes are tested in single- and two-phase flow models of fractured porous media using four example flow geometries. Results obtained for different fracture-matrix permeability ratios and, in the case of two-phase flow, different viscosity ratios are presented. Sealed fractures are also considered. A realistic fracture geometry is used to simulate water-flooding.

\section{Methodology}

In this section, an overview of the hybrid FEFV discretization schemes is presented. This is followed by a description of the set up of the numerical experiments employed in this study. The first scheme refers to the FEFVM in which both the fractures and the porous rock are 
discretized with the same element dimensionality. This is called FEFVM 2D or FEFVM 3D for 2D and 3D models, respectively. The second method unlike the previous scheme use lower dimensional elements for the fractures. This is called FEFVM 1D \& 2D or FEFVM 2D \& 3D for 2D and 3D models, respectively. The third scheme which uses the FEFVM with embedded discontinuity is accomplished by adding new nodes at the matrix-fracture interfaces (DFEFVM).

\subsection{Governing Equations}

Darcy's law, in its simplified form and ignoring gravitational forces, can be used to obtain velocity field,

$$
\mathbf{v}_{\mathbf{t}}=-k \lambda_{\mathrm{t}} \nabla p,
$$

where $\mathbf{v}_{\mathbf{t}}$ denotes the total velocity vector and $p$ represents the pressure values. The pressure equation for single- or two-phase flow in porous media in the absence of gravity and capillary forces can be written as

$$
\phi \frac{\partial p}{\partial t}=\nabla \cdot k \lambda_{\mathrm{t}} \nabla p+\dot{q}
$$

where $\dot{q}$ stands for external sinks and sources $\left[\mathrm{T}^{-1}\right]$. Note that for single-phase flow water saturation is equal to one and the total mobility is $\lambda_{t}=\frac{1}{\mu}$. Total mobility, $\lambda_{t}$, is multiplied with the permeability $k$, and depends on the viscosities $\mu_{\alpha}$, (where $\alpha=\mathrm{n}$, w) and relative permeability multipliers $k_{\mathrm{r} \alpha}$, for non-wetting (n) and wetting (w) phases.

$$
\lambda_{\mathrm{t}}=\left(\frac{k_{\mathrm{rn}}}{\mu_{\mathrm{n}}}+\frac{k_{\mathrm{rw}}}{\mu_{\mathrm{w}}}\right) .
$$

The calculation of $k_{\mathrm{r} \alpha}$ multipliers relies on experimentally parameterized non-linear models such as the Brooks and Corey (1964) or van Genuchten (1980) model. For pure advection the mass balance is given by,

$$
\phi \frac{\partial C}{\partial t}+\nabla \cdot\left(\mathbf{v}_{\mathbf{t}} C\right)=q .
$$

where $C$ denotes the concentration $\left[\mathrm{ml}^{-3}\right], t$ represents time, and $q$ is a mass-source/sink term $\left[\mathrm{ml}^{-3} \mathrm{~T}^{-1}\right]$. Buckley and Leverett (1942) showed that, in the absence of gravity and capillary forces, the position of the saturation front can be calculated as a product of $\mathbf{v}_{\mathbf{t}}$ and the fractional flow, $f_{\alpha}$, of the phase $\alpha$ of interest:

$$
f_{\alpha}=\frac{\lambda_{\alpha}}{\left(\lambda_{\mathrm{n}}+\lambda_{\mathrm{w}}\right)},
$$

where $\lambda_{\mathrm{n}}$ and $\lambda_{\mathrm{w}}$ are non-wetting and wetting phase mobilities, respectively. This leads to the closed form of the Buckley-Leverett equation:

$$
\phi \frac{\partial S_{\alpha}}{\partial t}+\nabla \cdot\left(f_{\alpha} \mathbf{v}_{\mathbf{t}}\right)-q_{\alpha}=0
$$

The saturation $S_{\alpha}[-]$ of phase $\alpha$ is defined as the ratio of its volume within the REV over the total pore volume. For two-phase flow, only one phase needs to be tracked because phase saturations always sum up to one. The Buckley-Leverett equation is a scalar conservation law associated with a non-convex saturation profile with a shock migrating with a speed equivalent to the slope of a line tangent to the fractional flow function, and originating at the 
irreducible saturation of the phase of interest (Bastian 1999). The non linearity arises due to the coupling of the pressure equation with the saturation equation via total mobility and the effects of saturation on velocity.

\subsection{Numerical Methods}

Similar to Huber and Helmig (1999) and Karimi-Fard and Firoozabadi (2003), we compute transient fluid pressure diffusion and miscible/immiscible displacement of compressible phases in a sequential manner: first, an elliptic-parabolic pressure equation is solved using the Bubnov-Galerkin finite element method, the flow velocity is obtained from the pressure gradient and Darcy's law. It is then used in the solution of the transport equation with a finite volume scheme. The idea of this FEFVM is to discretize the domain with finite elements and complementary finite volumes such that for each node of the finite element mesh a finite volume is created by connecting element barycentres via the midpoints of the associated edges (Paluszny et al. 2007). Therefore, finite volumes $v_{j}$ are constructed around the finite element nodes $n_{j}$. In contrast with Huber and Helmig (2000), we discretize permeability as piecewise constant from element to element so that the finite element conform to material interfaces.

There are two possibilities to discretize fractured porous media with the FEFVM (Juanes et al. 2002). The first option is to discretize the fractures with the same volumetric/surface finite elements as the rock matrix. The second option is to use lower dimensional surface/line elements for fractures. The first option requires the use of high aspect ratio-or very fine elements for fractures with small apertures. Since the nodes on either side of any fracture are shared, the method with lower dimensional fracture elements is incapable of simulating a system with a matrix permeability $\left(k_{\mathrm{m}}\right)$ higher than the fracture permeability $\left(k_{\mathrm{f}}\right)$.

Pressure Equation

In the Paluszny et al. (2007) approach, material properties such as permeability and porosity are constant within each element $e_{i}$. Using linear FE shape functions, $N_{i}$, the implicit space-time integration of Eq. 2 over the bounded domain $\Omega \subset R^{d}(d=2,3)$ for $\mathbf{x} \in \Omega$ yields,

$$
\begin{gathered}
{\left[\sum_{e} \int_{\Omega} \mathbf{N}^{\mathbf{T}} \phi \mathbf{N} d \mathbf{x}+\Delta t \sum_{e} \int_{\Omega} \nabla \mathbf{N}^{\mathbf{T}} \lambda_{\mathrm{t}} k \nabla \mathbf{N} d \mathbf{x}\right] \mathbf{p}^{t+\Delta t}} \\
=\left[\sum_{e} \int_{\Omega} \mathbf{N}^{\mathbf{T}} \phi \mathbf{N} d \mathbf{x}\right] \mathbf{p}^{t}+\Delta t \sum_{e} \int_{\Omega} \mathbf{N}^{\mathbf{T}} \dot{q} \mathbf{N} d \mathbf{x} .
\end{gathered}
$$

For lower dimensional fracture elements, the domain $\Omega$ consist of two overlapping domains, $\Omega_{\mathrm{f}}$ and $\Omega_{\mathrm{m}}$, as $\Omega_{\mathrm{m}} \subset R^{d}$ and $\Omega_{\mathrm{f}} \subset R^{d-1}(d=2,3)$. Equation 7 becomes,

$$
\begin{aligned}
& {\left[\sum_{e} \int_{\Omega_{\mathrm{m}}} \mathbf{N}^{\mathbf{T}} \phi \mathbf{N} d \mathbf{x}+\sum_{e} a_{i} \int_{\Omega_{\mathrm{f}}} \mathbf{N}^{\mathbf{T}} \phi \mathbf{N} d \mathbf{x}\right] \mathbf{p}^{t+\Delta t}} \\
& +\Delta t\left[\sum_{e} \int_{\Omega_{\mathrm{m}}} \nabla \mathbf{N}^{\mathbf{T}} \lambda_{t} k \nabla \mathbf{N} d \mathbf{x}+\sum_{e} a_{i} \int_{\Omega_{\mathrm{f}}} \nabla \mathbf{N}^{\mathbf{T}} \lambda_{t} k \nabla \mathbf{N} d \mathbf{x}\right] \mathbf{p}^{t+\Delta t}
\end{aligned}
$$


FEFV 1D\&2D

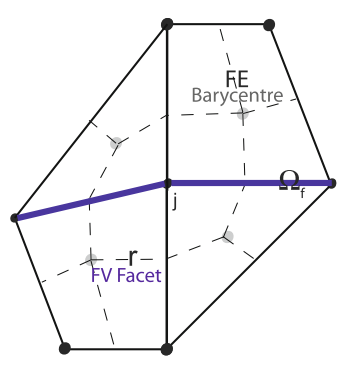

DFEFV 2D

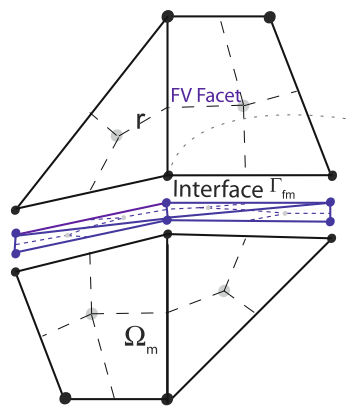

FEFV 2D

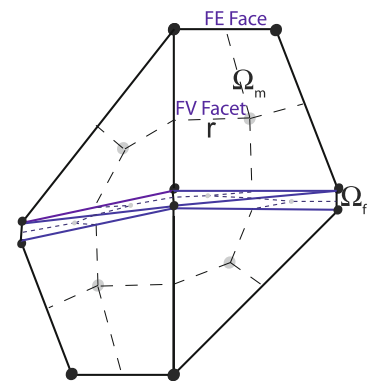

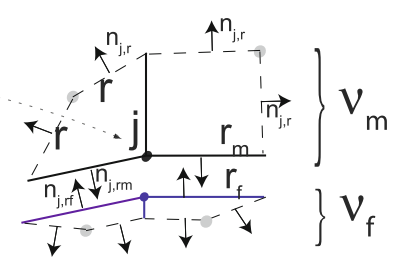

Fig. 1 Three different discretizations for the FEFV methods

$$
\begin{aligned}
& =\left[\sum_{e} \int_{\Omega_{\mathrm{m}}} \mathbf{N}^{\mathbf{T}} \phi \mathbf{N} d \mathbf{x}+\sum_{e} a_{i} \int_{\Omega_{\mathrm{f}}} \mathbf{N}^{\mathbf{T}} \phi \mathbf{N} d \mathbf{x}\right] \mathbf{p}^{t} \\
& +\Delta t\left[\sum_{e} \int_{\Omega_{\mathrm{m}}} \mathbf{N}^{\mathbf{T}} \dot{q} \mathbf{N} d \mathbf{x}+\sum_{e} a_{i} \int_{\Omega_{\mathrm{f}}} \mathbf{N}^{\mathbf{T}} \dot{q} \mathbf{N} d \mathbf{x}\right]
\end{aligned}
$$

where $a_{i}$ denotes the aperture of fracture element, $i$.

To embed discontinuities at material/finite element interfaces we develop a new hybrid method: DFEFVM. For this propose we locate new nodes at material interfaces so that they can represent concentration/saturation jump discontinuities at such locations in contrast to the continuous FEFVM (Fig. 1). Material interfaces exist where properties such as permeability, porosity, or relative permeability change abruptly, $\Gamma_{\mathrm{fm}} \in \Omega_{\mathrm{f}} \cap \Omega_{\mathrm{m}}$. The new nodes are introduced ascertaining that every node is member of only one sub-domain, either $\Omega_{\mathrm{f}}$ or $\Omega_{\mathrm{m}}$. To solve Eq. 2 with this DFEFVM we assume that the pressure of the wetting phase is continuous across these interfaces. Now, we adjust the global solution matrix arising from the pressure equation, to eliminate the extra unknowns due to the new nodes at the interfaces. This is explained through a simple one dimensional model containing two elements where node 2 is split for DFEFVM (Fig. 2). The element conductance matrices, $K^{1}$ and $K^{2}$, with the coefficients $a_{1}$ and $a_{2}$ for the finite elements 1 and 2 can be written as: 
Fig. 2 One dimensional model, containing two finite elements, discretized by the FEFVM and DFEVM

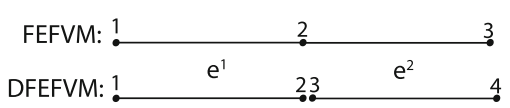

$$
\begin{aligned}
K^{1} & =\left[\begin{array}{cc}
a_{1} & -a_{1} \\
-a_{1} & a_{1}
\end{array}\right] \\
K^{2} & =\left[\begin{array}{cc}
a_{2} & -a_{2} \\
-a_{2} & a_{2}
\end{array}\right]
\end{aligned}
$$

The global conductance matrix for FEFVM reads,

$$
A=\left[\begin{array}{ccc}
a_{1} & -a_{1} & 0 \\
-a_{1} & a_{1}+a_{2} & -a_{2} \\
0 & -a_{2} & a_{2}
\end{array}\right]
$$

whereas for the DFEFVM it yields,

$$
A=\left[\begin{array}{cccc}
a_{1} & -a_{1} & 0 & 0 \\
-a_{1} & a_{1} & 0 & 0 \\
0 & 0 & a_{2} & -a_{2} \\
0 & 0 & -a_{2} & a_{2}
\end{array}\right]
$$

The second and third rows correspond to the nodes at the interface. Adding off-diagonal terms to couple the nodes 2 and 3 in matrix $A$ to the corresponding nodes from the same interface and modifying the diagonal one accordingly, gives,

$$
A=\left[\begin{array}{cccc}
a_{1} & -a_{1} & 0 & 0 \\
-a_{1} & a_{1}+a_{2} & 0 & 0-a_{2} \\
0-a_{1} & 0 & a_{2}+a_{1} & -a_{2} \\
0 & 0 & -a_{2} & a_{2}
\end{array}\right]
$$

This procedure forces the nodal pressure values on either side of the interface to become the same.

\section{Mass Balance Equation}

The finite volume approach is used to solve Eqs. 4 and 6. We employ piecewise constant $\mathrm{FV}$ interpolation functions, $M_{j}$ for each finite volume $v_{j}$, to integrate over the domain $V \in$ $V_{\mathrm{f}} \cup V_{\mathrm{m}}$. Applying the divergence theorem,

$$
\sum_{j} \int_{v} \mathbf{M} \phi \psi^{t+\Delta t} d V-\sum_{j} \int_{v} \mathbf{M} \phi \psi^{t} d V=\Delta t\left[\sum_{j} \oint_{\Gamma_{v}} \mathbf{F}^{t} \cdot \mathbf{n} d S+\sum_{j} \int_{v} \mathbf{M} q d V\right],
$$

where $\psi$ represents the primary variable (i.e., concentration and saturation), $\Gamma_{v}$ denotes the cell boundary, and $\mathbf{n}$ is the normal vector. For single-phase solute transport simulated with a first-order upwind scheme, the flux integral term of Eq. 4 for $v_{j}$ gives,

$$
\oint_{\Gamma_{v}} \mathbf{F}^{t} \cdot \mathbf{n} d S=\sum_{r}^{N_{r}}\left(H_{j, r} A_{j, r} \mathbf{n}_{j, r} \cdot \mathbf{v}_{\mathbf{t}} \psi_{\mathrm{u}}+\left(1-H_{j, r}\right) A_{j, r} \mathbf{n}_{j, r} \cdot \mathbf{v}_{\mathbf{t}} \psi_{\mathrm{c}}\right),
$$


where $\psi_{\mathrm{u}}$ denotes the primary variable of the upstream volume and $\psi_{\mathrm{c}}$ denotes the primary variable of the current volume, $\mathbf{n}_{j, r}$ is the normal vector of facet $r$ pointing outward of the finite volume $j$ and the function $H_{j, r}$ is defined as,

$$
H_{j, r}= \begin{cases}1 & \text { if } \mathbf{n} \cdot \mathbf{v}_{t}<0 \\ 0 & \text { otherwise. }\end{cases}
$$

There are $N_{r}$ facets $r$ of area $A_{j, r}$ in each finite volume $v_{j}$.

For the two-phase flow problem (Eq. 6) surface-integrated influxes are multiplied with $f_{\alpha i}^{t}\left(\psi_{\mathrm{u}}\right)$ evaluated with the upstream saturations. Outfluxes are multiplied with the $f_{\alpha i}^{t}\left(\psi_{\mathrm{c}}\right)$ of current volume. In any case, the fractional flow function of the current cell is used. So we have,

$$
\oint_{\Gamma_{\nu}} \mathbf{F}^{t} \cdot \mathbf{n} d S=\sum_{r}^{N_{r}}\left(H_{j, r} A_{j, r} \mathbf{n}_{j, r} \cdot \mathbf{v}_{\mathbf{t}} f_{\alpha i}\left(\psi_{\mathrm{u}}\right)+\left(1-H_{j, r}\right) A_{j, r} \mathbf{n}_{j, r} \cdot \mathbf{v}_{\mathbf{t}} f_{\alpha i}\left(\psi_{\mathrm{c}}\right)\right) .
$$

For the lower dimensional fracture elements, finite volume porosity is calculated as the sum of porosity of all sectors in $v$,

$$
\phi=\frac{\sum_{n=1}^{N_{\mathrm{sm}}} \phi_{\mathrm{m}} V_{\mathrm{sm}}}{\sum_{n=1}^{N_{\mathrm{sm}}} V_{\mathrm{sm}}}+\frac{\sum_{n=1}^{N_{\mathrm{sf}}} \phi_{\mathrm{f}} V_{\mathrm{fm}}}{\sum_{n=1}^{N_{\mathrm{sf}}} V_{\mathrm{fm}}},
$$

where $N_{\mathrm{sm}}$ and $N_{\mathrm{sf}}$ are the number of matrix and fracture finite volume sectors of the finite volume $v$, respectively, so that $N_{r}=N_{\mathrm{sm}}+N_{\mathrm{fm}}$. In 3D models, the parameter $V_{\text {sm }}$ denotes the volume of matrix finite volume sectors, and $V_{\text {sf }}$ is the surface of fracture finite volume sectors of the finite volume $v$.

Total velocity $\mathbf{v}_{\mathbf{t}}$ is calculated at the integration point of each finite volume facet using Eq. 1. Consequently fluxes are continuous across the finite volume facets. For the truncated finite volumes at the outflow boundaries $\Gamma_{\text {out }}$, however, we calculate $\mathbf{v}_{\mathbf{t}}$ for the Dirichlet boundary conditions via,

$$
\sum_{\Gamma_{\text {out }}} A_{j, r} \mathbf{n}_{j, r} \cdot \mathbf{v}_{\mathbf{t}}=-\sum_{\Gamma_{v}} A_{j, r} \mathbf{n}_{j, r} \cdot \mathbf{v}_{\mathbf{t}}
$$

After splitting the FE mesh at the material interfaces, we treat these as internal boundaries when solving the transport equation. Therefore, for the DFEFVM, an approximation of the flux at the internal boundaries $\Gamma_{\mathrm{fm}}$ between fractures and matrix is required. Equation 13 is employed to calculate these fluxes. For each finite volume in a continuous space, flux continuity is obtained if,

$$
\sum_{\Gamma_{v}} A_{j, r} \mathbf{n}_{j, r} \cdot \mathbf{v}_{\mathbf{t}}=0
$$

At the fracture-matrix interfaces, finite volumes $v_{\mathrm{m}}$ and $v_{\mathrm{f}}$ as $v \in v_{\mathrm{m}} \cup v_{\mathrm{f}}$ are truncated by boundaries $r \cup r_{\mathrm{m}}$ and $r \cup r_{\mathrm{f}}$, respectively. In order to evaluate Eqs. 10 or 11, the unknown fluxes, $\mathbf{n}_{j, r_{\mathrm{m}}} \cdot \mathbf{v}_{\mathbf{t}}^{*}=-\mathbf{n}_{j, r_{\mathrm{f}}} \cdot \mathbf{v}_{\mathbf{t}}^{*}$, at the interface $\Gamma_{\mathrm{fm}}$ need to be found. The $\mathbf{n}_{j, r_{\mathrm{m}}}$ and $\mathbf{n}_{j, r_{\mathrm{f}}}$ are the normal vectors to the outward facing elements faces on matrix and fracture sides, respectively. Since $\mathbf{v}_{\mathbf{t}}$ is discontinuous at these finite element faces a $\mathbf{v}_{\mathbf{t}}^{*}$ has to be calculated by rewriting the Eq. 14 for the truncated finite volumes, $v_{\mathrm{m}}$ and $v_{\mathrm{f}}$ from,

$$
\sum_{\Gamma_{\nu_{\mathrm{m}}}} A_{j, r} \mathbf{n}_{j, r} \cdot \mathbf{v}_{\mathbf{t}}+\sum_{\Gamma_{\mathrm{fm}}} A_{j, r_{\mathrm{m}}} \mathbf{n}_{j, r_{\mathrm{m}}} \cdot \mathbf{v}_{\mathbf{t}}^{*}=-\sum_{\Gamma_{v_{\mathrm{f}}}} A_{j, r} \mathbf{n}_{j, r} \cdot \mathbf{v}_{\mathbf{t}}-\sum_{\Gamma_{\mathrm{fm}}} A_{j, r_{\mathrm{f}}} \mathbf{n}_{j, r_{\mathrm{f}}} \cdot \mathbf{v}_{\mathbf{t}}^{*} .
$$


This can not be used to calculate $\mathbf{v}_{\mathbf{t}}^{*}$ for the finite volumes which contain both internal boundaries $\left(\Gamma_{\mathrm{fm}}\right)$ and outflow boundaries $\left(\Gamma_{\mathrm{out}}\right)$. For these finite volumes we calculate $\mathbf{v}_{\mathbf{t}}^{*}$ by taking the average of $\mathbf{v}_{\mathbf{t}}$ at each side of the interface $\Gamma_{\mathrm{fm}}$.

Similar to Durlofsky (1998), in this analysis we use the implicit pressure explicit saturation (IMPES) formulation to solve the two-phase flow equations. This formulation has been used commonly as it provides a simple and efficient implementation (Chen et al. 2006). However, this formulation suffers from instability and time step limitation specially for the model containing small elements (Matthäi et al. 2010). The scheme is implemented in and the equations are solved using CSMP++ which is an object-oriented application programmer interface (API), designed for the simulation of complex subsurface processes and their interactions (Matthäi et al. 2007; Zaretskiy et al. 2010; Geiger et al. 2010; Unsal et al. 2010; Paluszny and Zimmerman 2011).

\subsection{Model Configuration for the Numerical Experiments}

Five models are used to evaluate the described alternative FEFV discretization approaches, see Table 1. These models are described in the following.

\section{Model 1}

Cross-sectional model of a single fracture $(17.5 \mathrm{~cm}$ long) connected only to the right boundary of the $20 \times 1 \mathrm{~cm}$ model domain is used (Fig. 3), meshed with triangular elements. In the experiments, tracer enters through the left boundary (Dirichlet boundary condition), driving by a far field pressure difference of $1 \times 10^{3} \mathrm{~Pa}$ between the left and the right boundaries. We use a permeable porous media, $k_{\mathrm{m}}=4 \times 10^{-12} \mathrm{~m}^{2}$, for the matrix with porosity of 0.3 . The fracture has an aperture of $1 \mathrm{~mm}$ with the permeability of $4 \times 10^{-10} \mathrm{~m}^{2}$ and porosity of one. Table 2 lists three level of mesh refinement details.

Model 2

Test model for an immiscible displacement in a $20 \times 1 \mathrm{~cm}$ rock sample has a single throughgoing fracture with the constant aperture size of $1 \mathrm{~mm}$. Fracture and matrix porosities are the

Table 1 Material properties of the test models (Figs. 3, 4, 5, 6, and 13 )

\begin{tabular}{lllllll}
\hline Parameter & Units & Model 1 & Model 2 & $\begin{array}{l}\text { Single sealed } \\
\text { fracture model }\end{array}$ & $\begin{array}{l}\text { Flow impediment } \\
\text { model }\end{array}$ & $\begin{array}{l}\text { Bristol channel } \\
\text { model }\end{array}$ \\
\hline Dimensions & $\mathrm{cm}$ & $20 \times 1$ & $20 \times 1$ & $100 \times 20$ & $100 \times 100$ & $300 \times 300$ \\
$\phi_{\mathrm{m}}$ & - & 0.3 & 0.3 & 0.3 & 0.3 & 0.3 \\
$\phi_{\mathrm{f}}$ & - & 1 & 1 & 0.1 & 0.3 & 0.3 \\
$k_{\mathrm{m}}$ & $\mathrm{m}^{2}$ & $4 \times 10^{-12}$ & $4 \times 10^{-15}$ & $1 \times 10^{-12}$ & $1 \times 10^{-14}$ & $1 \times 10^{-14}$ \\
$k_{\mathrm{f}}$ & $\mathrm{m}^{2}$ & $4 \times 10^{-10}$ & $4 \times 10^{-10}$ & $1 \times 10^{-18}$ & $1 \times 10^{-17}$ & $4 \times 10^{-11}$ \\
\hline
\end{tabular}

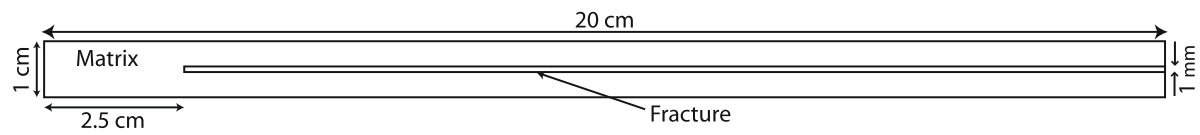

Fig. 3 Single fracture, Model 1 
Table 2 Mesh parameters of Model 1 for three level of refinements (Fig. 8)

\begin{tabular}{lllll}
\hline Mesh & $\begin{array}{l}\text { Element size } \\
(\mathrm{mm})\end{array}$ & $\begin{array}{l}\text { Nodes } \\
\text { FEFVM 1D \& 2D }\end{array}$ & $\begin{array}{l}\text { Nodes } \\
\text { FEFVM 2D }\end{array}$ & $\begin{array}{l}\text { Nodes } \\
\text { DFEFVM }\end{array}$ \\
\hline 1 & 2.5 & 415 & 493 & $493+150$ \\
2 & 1 & 2607 & 2330 & $2330+342$ \\
3 & 0.5 & 8242 & 8397 & $8397+710$ \\
\hline
\end{tabular}

Table 3 Element size and the number of nodes in Model 2 for different schemes

\begin{tabular}{lllll}
\hline Mesh & $\begin{array}{l}\text { Element size } \\
(\mathrm{mm})\end{array}$ & $\begin{array}{l}\text { Nodes } \\
\text { FEFVM 1D \& 2D }\end{array}$ & $\begin{array}{l}\text { Nodes } \\
\text { FEFVM 2D }\end{array}$ & $\begin{array}{l}\text { Nodes } \\
\text { DFEFVM }\end{array}$ \\
\hline 1 & 2.5 & 414 & 507 & $507+170$ \\
\hline
\end{tabular}

Fig. 4 Three triangular element meshes (1-3) with increasing levels of uniform refinement (single sealed fracture model)

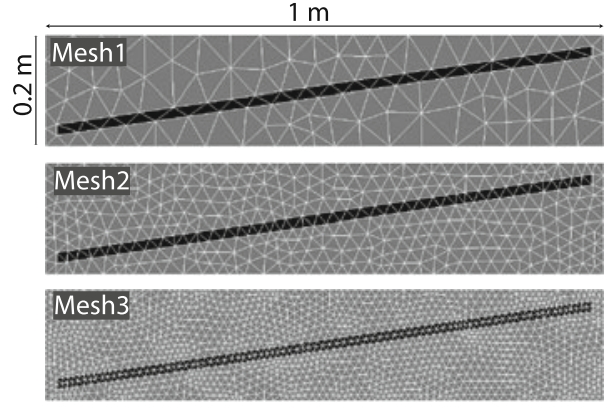

same as in model 1 . A permeability of $4 \times 10^{-15}$ and $4 \times 10^{-10} \mathrm{~m}^{2}$ are assigned to the rock matrix and fracture, respectively. The Brooks-Corey relative permeability model,

$$
\begin{aligned}
& k_{\mathrm{rw}}=S^{\frac{2+3 \lambda}{\lambda}} \\
& k_{\mathrm{rn}}=(1-S)^{2}\left(1-S^{\frac{2+\lambda}{\lambda}}\right),
\end{aligned}
$$

with a $\lambda$ value of 2 is used for both the fracture and the matrix. Triangles are used to discretize the model (Table 3). The model is initially saturated with oil, then water is injected through the left boundary driven by the applied pressure gradient of $5 \times 10^{3} \mathrm{pa} / \mathrm{m}$.

\section{Single Sealed Fracture Model}

We study the convergence of the method on a $2 \mathrm{D}$ domain with a size of $1 \times 0.2 \mathrm{~m}$. The model has a single sealed diagonal fracture with a permeability of $10^{-18}$ and $10^{-12} \mathrm{~m}^{2}$ for the fracture and the matrix, respectively. The sealed fracture and matrix porosity are set to 0.1 and 0.3 , respectively. Five triangular element meshes (1-5) with increasing levels of uniform refinement were used to further quantify mesh dependence of the results and mesh convergence. Figure 4 shows three mesh refinements used in the convergence study. A tracer plume is injected for $30 \mathrm{~h}$ from the left boundary driven by a horizontal pressure gradient of $1 \times 10^{3} \mathrm{~Pa} / \mathrm{m}$. 
Fig. 5 Triangulation for the sealed fracture network

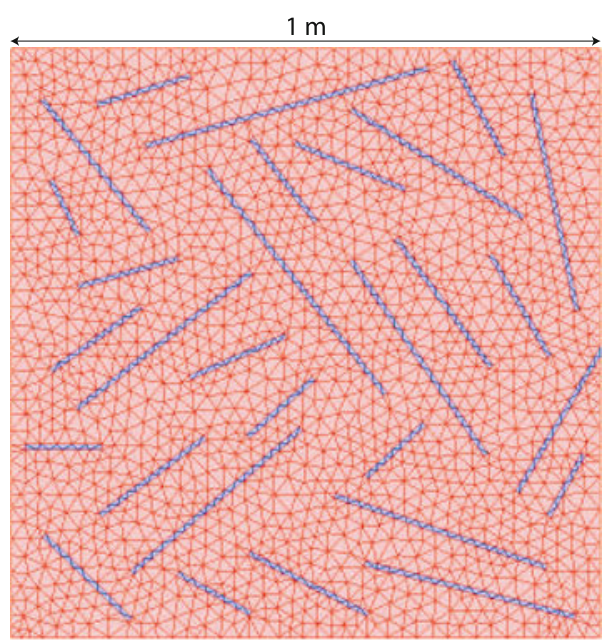

Flow Impediment Model

A 2D horizontal domain (Fig. 5) with a size of $1 \times 1 \mathrm{~m}$ is employed to compare the alternative numerical approaches for modeling single-phase flow. This model contains variably oriented separate flow impediments with a width of $1 \mathrm{~cm}$. The model is initially filled with water, then a tracer is injected through the top boundary for 10days. The tracer is then replaced with fresh water through continuous injection. Flow is driven by a constant pressure gradient of $1.9 \times 10^{4} \mathrm{~Pa} / \mathrm{m}$. The rock matrix and impediments have a permeability of $1 \times 10^{-14}$ and $1 \times 10^{-17} \mathrm{~m}^{2}$, respectively. Both flow-domains have the same porosity equal to 0.3 .

\section{Bristol Channel Model}

A $3 \times 3 \mathrm{~m}$ cross-sectional model of a fractured limestone bed mapped at the Bristol Channel coast, UK, (Belayneh 2004) is discretized by triangular elements (Fig. 6; Table 1). This model contains a set of well-interconnected fractures. The fracture matrix permeability contrast is set to $4 \times 10^{3}$ with the uniform fracture aperture of $1 \mathrm{~cm}$. A linear relative permeability model is assigned to the fractures and the Brooks-Corey model with the Brooks-Corey parameter of 2 is used for the rock matrix. A pressure difference of $1 \times 10^{3} \mathrm{~Pa}$ is prescribed between the top and the bottom boundaries (Fig. 7). Initially, the model is saturated with oil. The water injector is located at the top boundary.

\section{Results}

In this section, the accuracy and performance of the different hybrid discretization schemes (FEFVM 2D, FEFVM 1D \& 2D, and DFEFVM) are illustrated through numerical experiments. We start with the single fracture model comparing the schemes' performance for single-phase flow. One aspect of this analysis is the effect of fracture matrix permeability contrast on the accuracy of the results. For two-phase flow a simple single fracture model is considered. The aim is to measure the error of the different schemes for different fluid viscosity ratios. Furthermore, the single sealed fracture model and flow impediment model are 


\section{D\&2D elements}

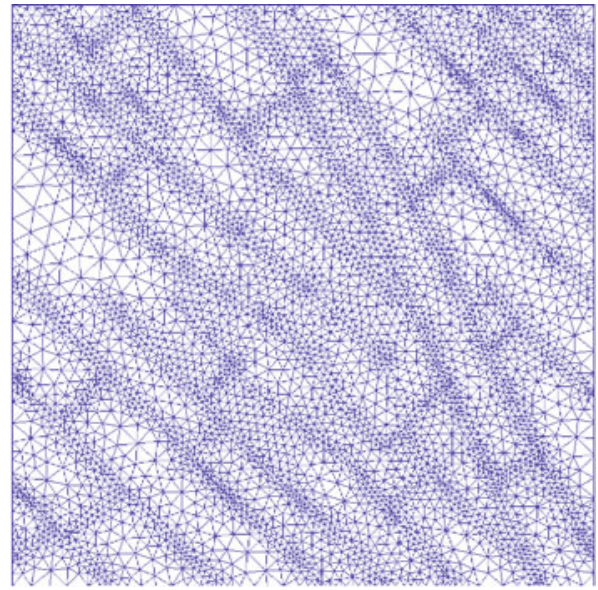

\section{$2 \mathrm{D}$ elements}

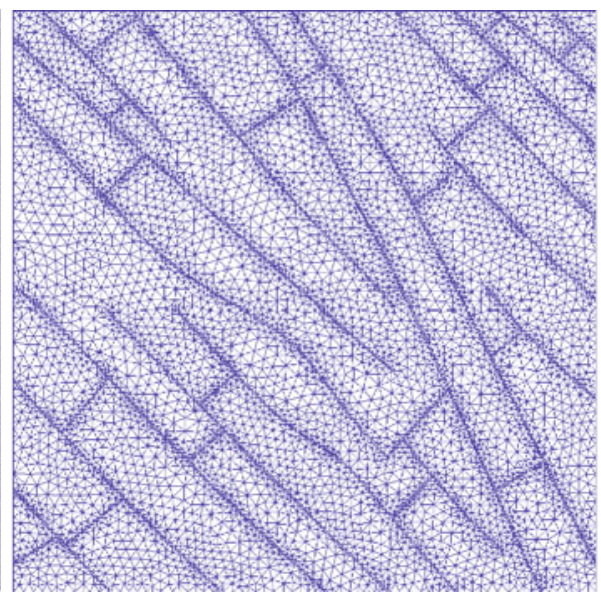

Fig. 6 Two different discretizations of the fractured limestone bed
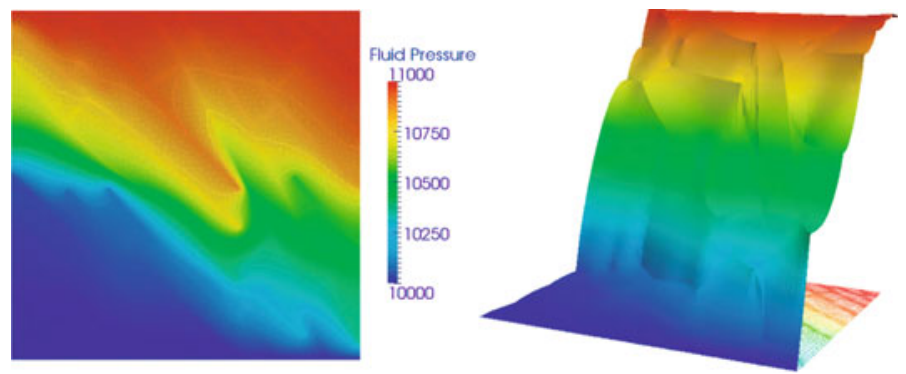

Fig. 7 Calculated pressure field after $1 \mathrm{~min}$ for the fractured limestone bed. The right plot illustrates the pressure field in 3D which presents the pressure changes inside the fractures

used to study the performance of the numerical schemes for a tracer advection. The section concludes with a comparison among the different schemes in water flooding simulation with the Bristol channel model.

\subsection{Single Phase Flow in a Single Fracture (Model 1)}

The first test is to evaluate the three schemes for different levels of mesh refinement. Figures 8 and 9 show the concentration fronts after 70 and $720 \mathrm{~s}$ of injection for three level of mesh refinements. The unphysical lateral smearing seen in the two continuum FEFV methods be attributed to the fact that the finite volumes at the fracture matrix interfaces contain both fracture and matrix regions (Fig. 8). This retards solute transport in the fracture whereas the concentration front in the fracture as computed using DFEFVM moves with the speed corresponding to the flow velocity of the fracture. The discrepancy between the tracer velocity of FEFV methods and DFEFVM decreases with decreasing mesh size refinement. However, a difference exists even for a very fine mesh (Fig. 9). This implies that in the case of FEFV methods concentration advance in higher permeability regions is slower than what expected.

Breakthrough curves are commonly used to describe transport behavior of heterogeneous porous media. Figure 10 shows the resulting breakthrough curves of the numerical schemes. 
Fig. 8 Three mesh refinements and the result of concentration front for three schemes at $t=70$ s. (a) FEFVM 1D \& 2D, (b) FEFVM 2D, and (c) DFEFVM
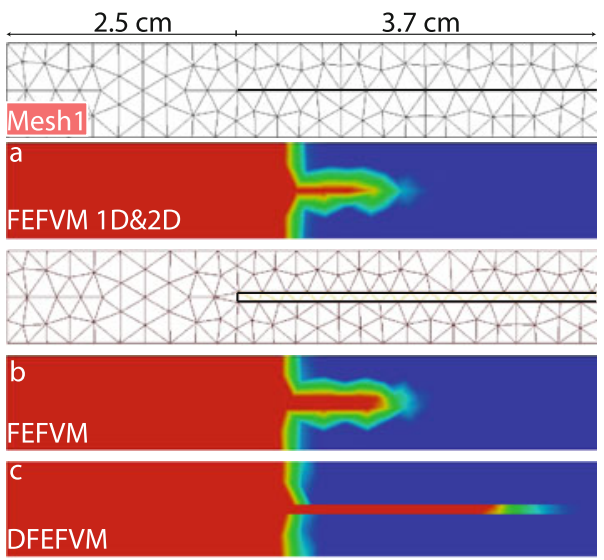
$2.5 \mathrm{~cm}$ $3.7 \mathrm{~cm}$
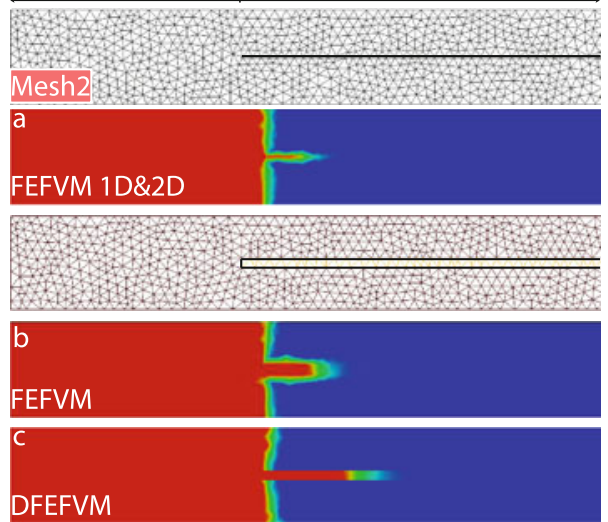

$2.5 \mathrm{~cm}$ $3.7 \mathrm{~cm}$
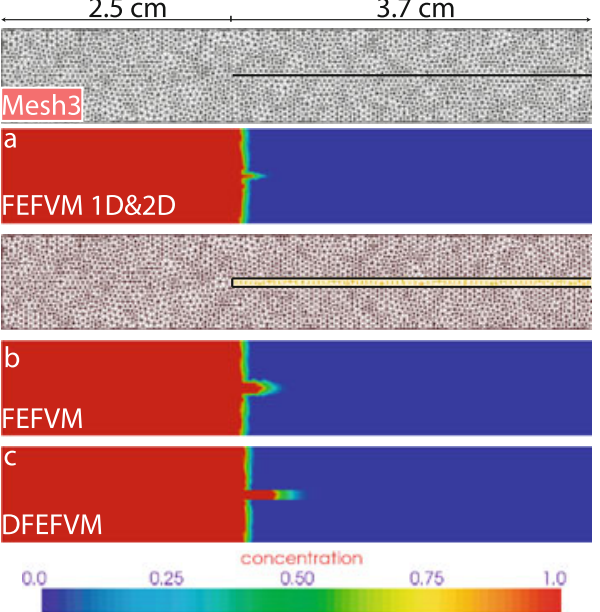

Two concentration jumps in breakthrough curves are expected. The first jump corresponds to when the tracer passing through the fracture reaches the right boundary. The next one occurs as the whole domain becomes infiltrated by the tracer. DFEFVM captures two jumps 
Fig. 9 Concentration fronts at $t=720 \mathrm{~s}$ for different mesh refinements and numerical schemes. (a) FEFVM 1D \& 2D,

(b) FEFVM 2D, and (c)

DFEFVM
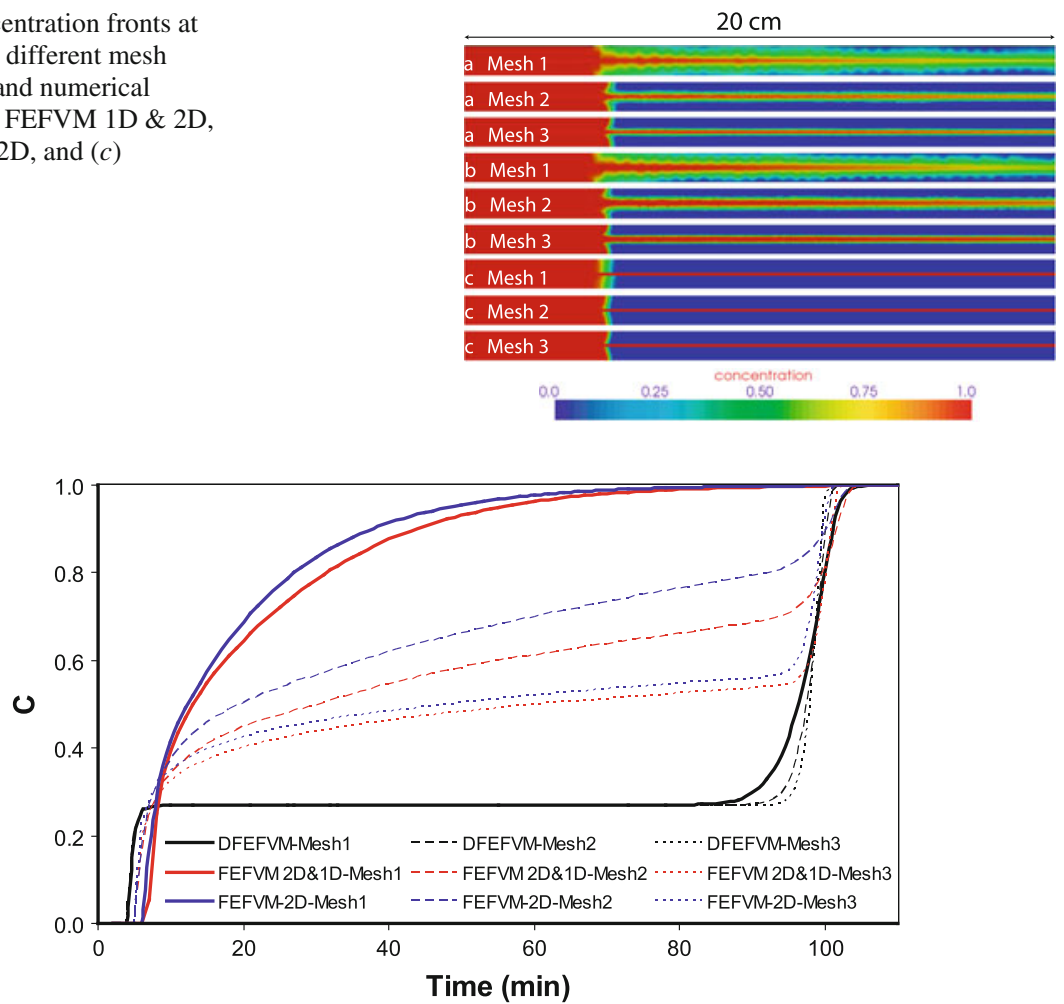

Fig. 10 Breakthrough curves for three different mesh refinements and numerical schemes

with minor numerical dispersion while both continuum FEFV approaches only show these for the highly refined meshes (Fig. 10). Interestingly, for the coarsest mesh, the continuum approaches fails to reveal any jumps. They produce considerable errors even for the finest mesh size. The jumps arrive at the outlet after 5 and $98 \mathrm{~min}$, respectively. These can be used to calculate the error of each solution in the breakthrough curves. We calculate two error norms, $L^{1}$ and $L^{2}$, for studying the accuracy of these schemes. Table 4 shows the concentration discretization error for the three numerical approaches as evaluated for the three levels of mesh refinement. The FEFVM combined with the lower dimensional fracture approach gives better results than the FEFVM for single phase flow. This was already argued by Juanes et al. (2002). The DFEFVM at the coarsest mesh solution already is more precise than the FEFVM on the finest mesh (Table 4). The number of unknowns for the DFEFVM at the coarsest mesh is almost 13 times less than that for the FEFVM at the finest mesh.

\subsubsection{Effect of Permeability Contrast}

The effect of fracture-matrix permeability contrast on the performance of each scheme is studied by conducting simulations on Mesh 3. Variations in permeability are achieved by retaining the permeability of the fracture, i.e., $k_{\mathrm{f}}=4 \times 10^{-10}$, but altering the permeability of matrix, i.e., $k_{\mathrm{m}}=4 \times 10^{-11}, 4 \times 10^{-12}, 2 \times 10^{-12}$, and $4 \times 10^{-13}$. Figure 11 depicts the concentration fronts obtained with the three approaches. The tracer fills the fracture rapidly for all cases shown in Fig. 11, however, it advances the fastest in the DFEFVM model. 
Table 4 Discretization errors for single-phase flow as a function of mesh refinement (Model 1)

\begin{tabular}{|c|c|c|c|c|c|c|}
\hline \multirow[b]{2}{*}{ Mesh } & \multicolumn{3}{|l|}{$L^{1}$} & \multicolumn{3}{|l|}{$L^{2}$} \\
\hline & $\begin{array}{l}\text { FEFVM } \\
1 \mathrm{D} \& 2 \mathrm{D}(\%)\end{array}$ & $\begin{array}{l}\text { FEFVM } \\
2 \mathrm{D}(\%)\end{array}$ & $\begin{array}{l}\text { DFEFVM } \\
2 \mathrm{D}(\%)\end{array}$ & $\begin{array}{l}\text { FEFVM } \\
1 \mathrm{D} \& 2 \mathrm{D}(\%)\end{array}$ & $\begin{array}{l}\text { FEFVM } \\
2 \mathrm{D}(\%)\end{array}$ & $\begin{array}{l}\text { DFEFVM } \\
\text { 2D }(\%)\end{array}$ \\
\hline 1 & 111.61 & 115.45 & 4.49 & 12.13 & 12.49 & 1.40 \\
\hline 2 & 58.55 & 72.60 & 2.79 & 6.30 & 7.87 & 1.24 \\
\hline 3 & 40.56 & 43.85 & 2.15 & 4.35 & 4.68 & 1.21 \\
\hline
\end{tabular}

Mesh 1 is the coarsest and Mesh 3 the finest

Fig. 11 Concentration fronts for the three fracture-matrix permeability contrasts at different time (Mesh 3). (a) FEFVM 1D \& 2D, (b) FEFVM 2D, and (c) DFEFVM

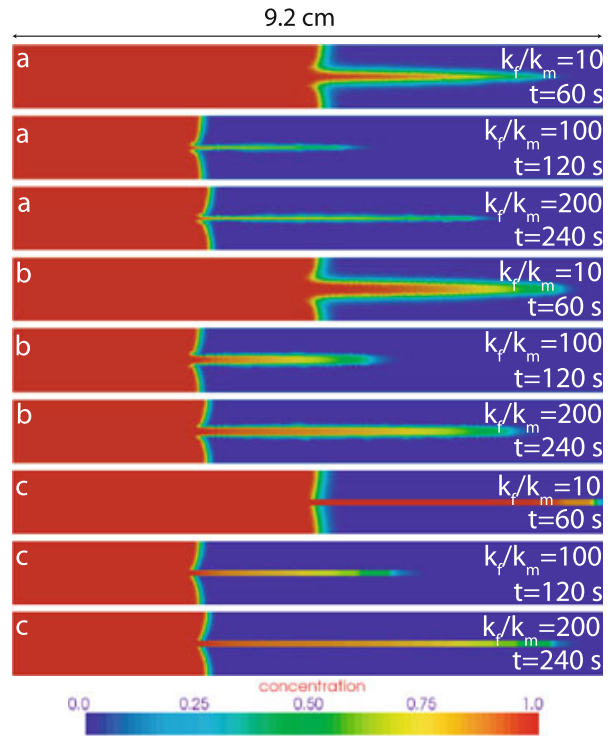

Table 5 Relative $L^{2}$ norms for the four different fracture-matrix permeability ratios applied to Model 1

\begin{tabular}{llll}
\hline $\begin{array}{l}\text { Permeability } \\
\text { ratio } k_{\mathrm{f}} / k_{\mathrm{m}}\end{array}$ & $\begin{array}{l}\text { FEFVM } \\
\text { 1D \& 2D }(\%)\end{array}$ & $\begin{array}{l}\text { FEFVM } \\
\text { 2D }(\%)\end{array}$ & $\begin{array}{l}\text { DFEFVM } \\
\text { 2D }(\%)\end{array}$ \\
\hline 10 & 21.44 & 21.11 & 1.00 \\
100 & 11.59 & 10.77 & 2.99 \\
200 & 21.22 & 19.31 & 3.97 \\
1000 & 23.70 & 21.46 & 5.00 \\
\hline
\end{tabular}

This discrepancy is most pronounced for the case with the lowest fracture-matrix permeability ratio. Breakthrough curves are plotted in Fig. 12 for all three cases. The differences are considerable, as shown in Table 5. The $L^{2}$ error is larger for the higher fracture-matrix permeability contrasts. 


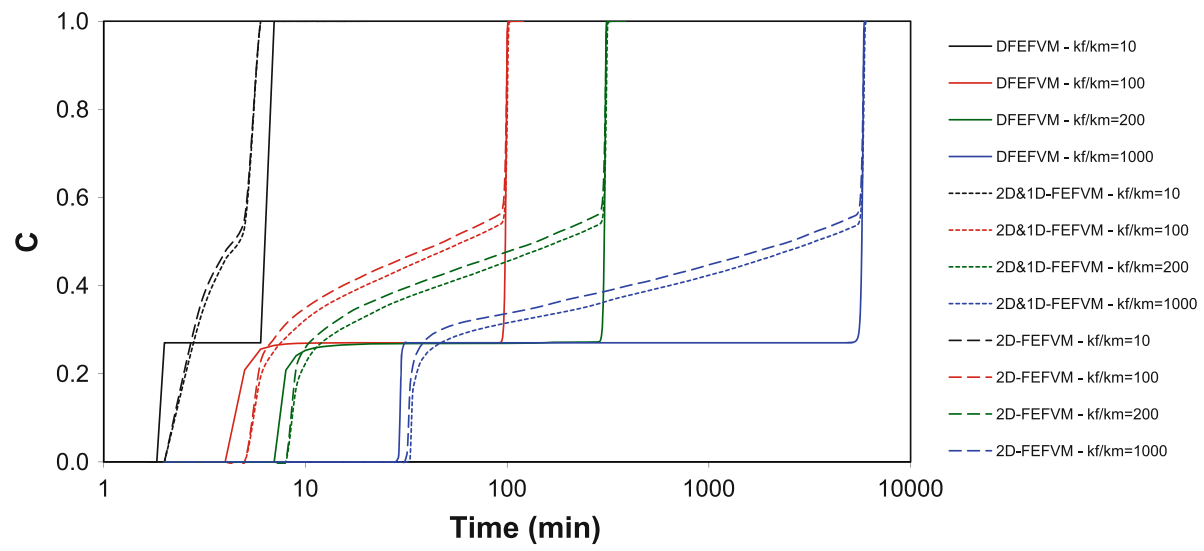

Fig. 12 Comparison of breakthrough curves for four different fracture-matrix permeability ratios calculated by three numerical schemes

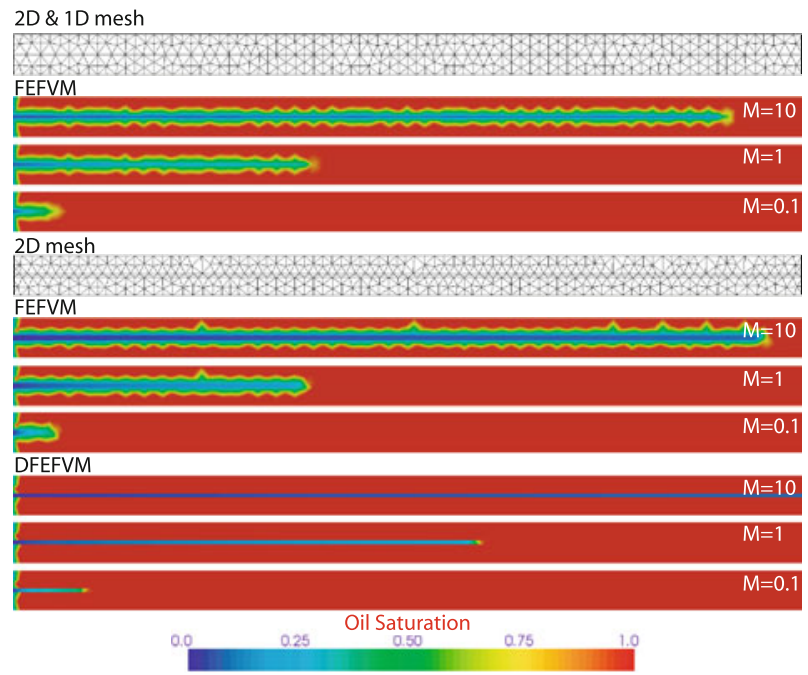

Fig. 13 Saturation fronts calculated by three numerical schemes for different viscosity ratios at $t=60 \mathrm{~s}$, (Model 2)

\subsection{Two-Phase Flow in a Single Fracture}

Model 2 is simulated with different viscosity $\operatorname{ratios}\left(M=\mu_{\text {displacing phase }} / \mu_{\text {displaced phase }}\right)$ to assess how the three discretization schemes handle water flooding. This is achieved by retaining a constant viscosity for the water of $0.001 \mathrm{~Pa}$ but varying the viscosity of the oil from 0.0001 , to 0.001 , to $0.01 \mathrm{~Pa}$ s (Fig. 13). The $S_{\mathrm{W}}$ at the shock front is the highest for the lowest viscosity ratio. Self-sharpening of the front is most pronounced for the smallest $M$ because the velocity in front of the shock is much smaller than that behind the shock. The DFEFVM yields earlier water breakthrough than the FEFVM. For a viscosity ratio of 0.1 , water breakthrough occurs in less than a minute in the DFEFVM model, but takes about 2 min with the FEFVM. The absolute difference in breakthrough time is maximized at $M=10$ 

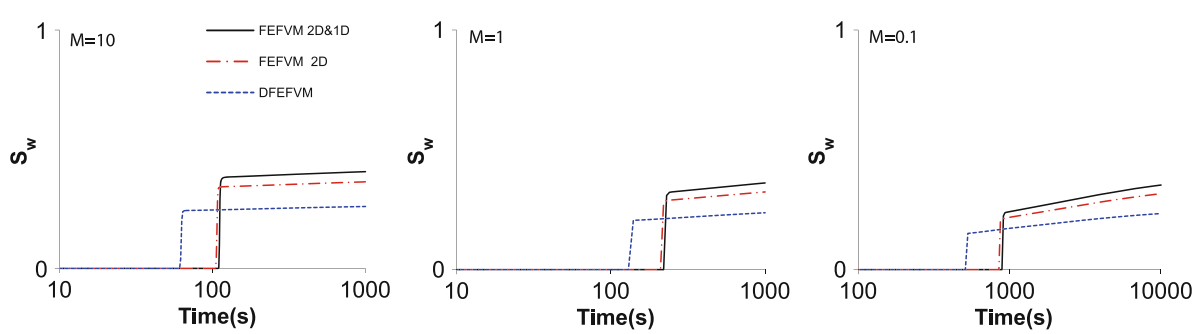

Fig. 14 Comparison of water saturation breakthrough curves calculated by three numerical schemes for different viscosity ratios (Model 2)
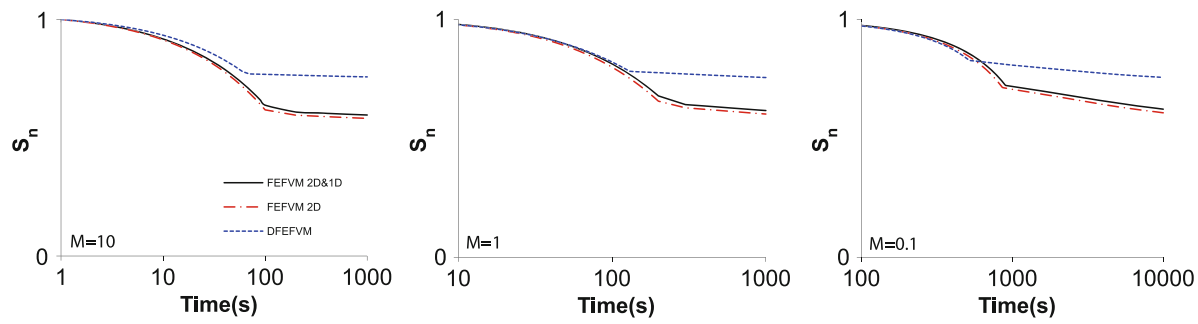

Fig. 15 Total oil saturation curves for different viscosity ratios (Model 2)
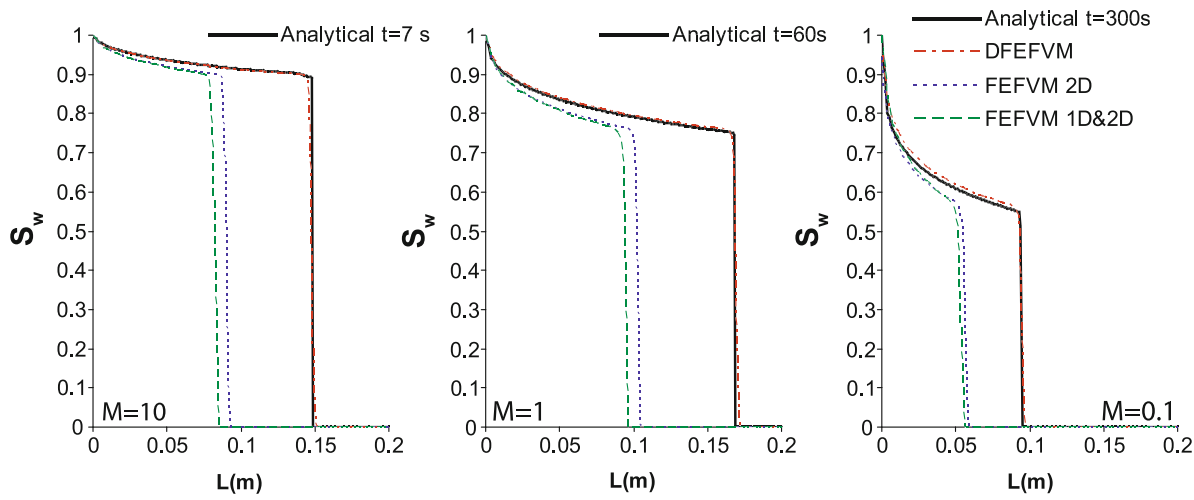

Fig. 16 Comparison of DFEFVM and FEFVM against analytical solution for $M=0.1,1$, and 10 . The water saturation profiles are measured at the middle of the fracture in Model 2

(Fig. 14). For all viscosity ratios the results of the continuum methods are similar. Total oil saturation versus time is plotted in Fig. 15. Here, the continuum models yield unrealistic high oil recoveries. A first order dependence on discretization of the fracture matrix interface causes this unsatisfactory feature. The DFEFVM reveals its superiority to the FEFV methods by eliminating this effect. It has the advantage that it allows evaluating the fractional flow without averaging nodal saturation values of elements with different relative permeability relationships. In order to compare results with an analytical solution, we apply a constant influx at the left boundary and use the velocity calculated at the first time step for the entire simulations. Note that the analytical solution is only solved for the fracture domain since the permeability of the matrix is very low. The results of the DFEFVM are in a good agreement with the analytical solution, but the shock fronts calculated with the FEFVM move more slowly (Fig. 16). 

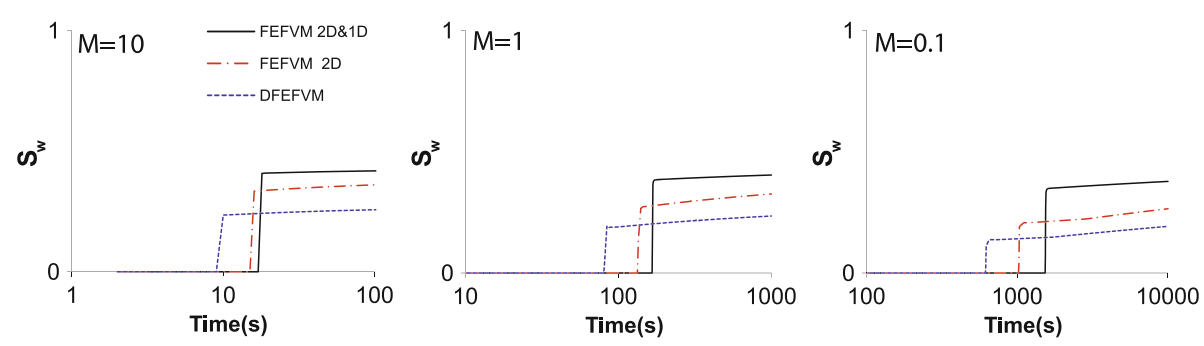

Fig. 17 Comparison of water saturation breakthrough curves calculated by three numerical schemes for different viscosity ratios (Model 2). The $\lambda$ value of 0.2 and 10 are assigned for the fracture and matrix, respectively
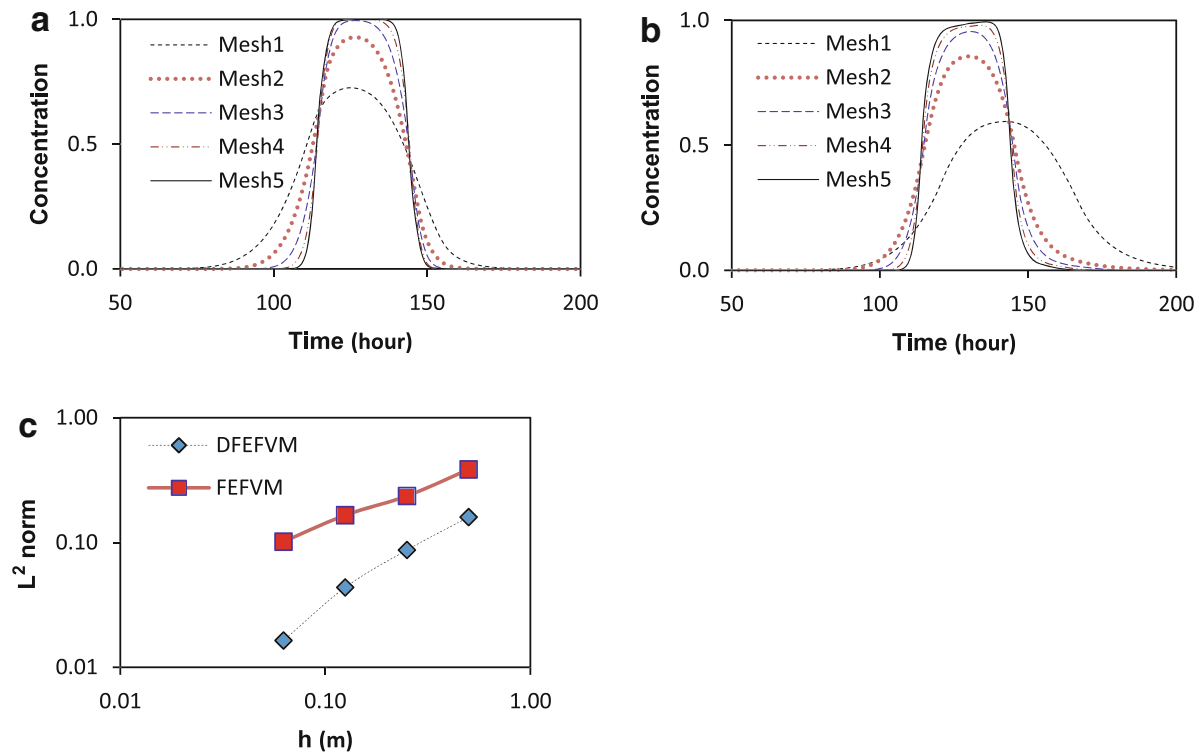

Fig. 18 Breakthrough curves for single sealed fracture model for different mesh refinements; a DFEFVM, b FEFVM, and c $L^{2}$ errors versus element sizes $(h)$ for different mesh refinements

Furthermore, the Model 2 is simulated with different Brooks-Corey parameter. The $\lambda$ value of 0.2 and 10 are assigned for the fracture and matrix, respectively. Figure 17 reveals the water saturation breakthrough curves. The FEFVM combined with the lower dimensional fracture approach (FEFVM 1D \& 2D) produces less accurate results than the FEFVM.

\subsection{Single Sealed Fracture Model—Convergence Study}

As explained earlier, sealed fractures or faults with a lower transmissivity than the country rock cannot be represented using lower dimensional elements. Therefore, we only compare the FEFVM 2D with the DFEFVM. The breakthrough curves for different mesh refinements show the effect of mesh refinement on the results (Fig. 18a, b). The $L^{2}$ errors are plotted against element size $(h)$ in Fig. 18c. The $L^{2}$ norms are calculated as $\sqrt{\sum\left(c-c_{r}\right)^{2}}$, where $c_{r}$ is the result of FEFVM 2D on Mesh 5 for FEFVM 2D, and the result of DFEFVM on Mesh 5 for DFEFVM. It is evident that the convergence order is higher for the DFEFVM, and the DFEFVM $L^{2}$ errors are much smaller than the errors of the FEFVM. 

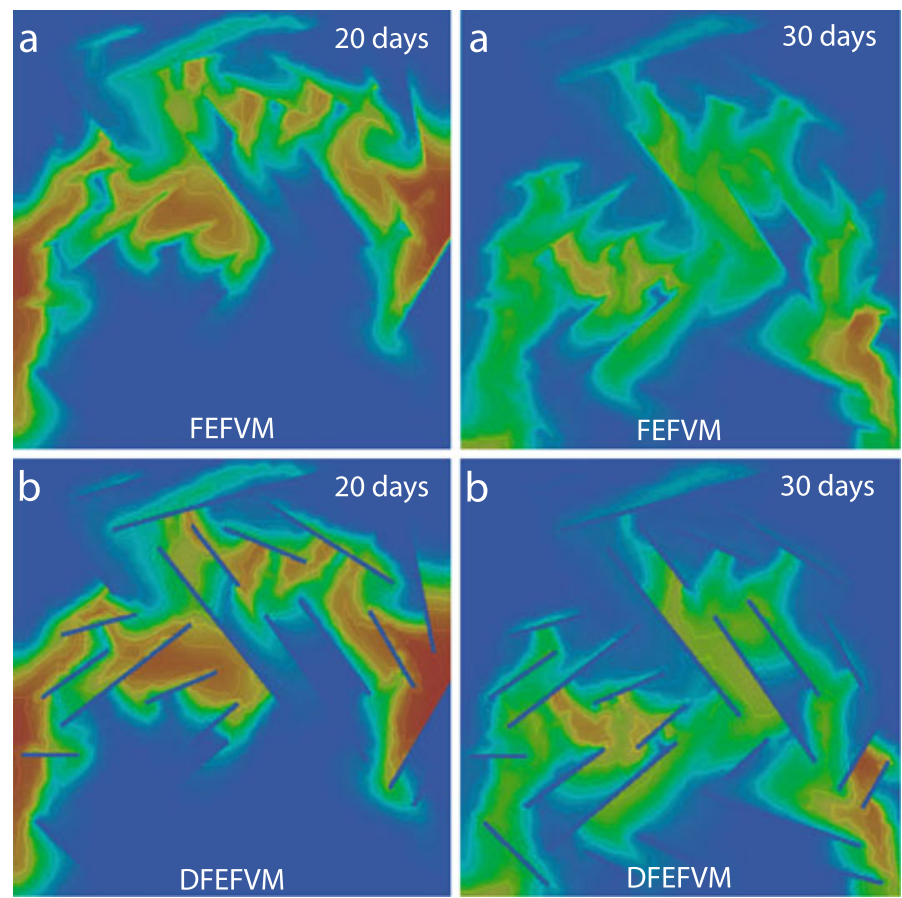

Concentration

0.0

0.5

1.0

Fig. 19 Concentration fronts after 20 and 30 days for flow impediment model. Two different schemes, DFEFVM and FEFVM, are compared. a FEFVM and b DFEFVM

\subsection{Flow Impediment Model}

Similar to the previous example, this section only focuses on comparing the FEFVM 2D with the DFEFVM. Figure 19 shows tracer distributions after 20 and 30 days. The flow impediments influence the pressure and velocity fields in both schemes. The velocities are identical for the FEFVM and DFEFVM, but the solute transport differs behavior (Figs. 19 and 20). The FEFVM is more dispersive, see Fig. 21. Furthermore, the total mass in time is shown in Fig. 22. In spite of the equal input mass flux for both methods, the output mass flux calculated by the DFEFVM is higher than that of FEFVM 2D. The FEFVM 2D yields a more dispersive result.

\subsection{Water Flooding of the Bristol Channel Limestone Model}

To further illustrate the advantages of the DFEFVM, a two dimensional model of Bristol Channel limestone model is used to conduct a water flooding experiment. Saturation fronts at different time steps are plotted in Fig. 23. Due to the low permeability of the host rock, the flow is channelized in the fractures. However, ultimate recovery is controlled by the properties of the rock matrix. Also, there is a discrepancy of almost 4 days between water breakthrough predicted with the FEFVM and the DFEFVM (Fig. 24). The amount of recovered oil is higher for the FEFVM due to the incorrect oil displacements at the fracture-matrix 


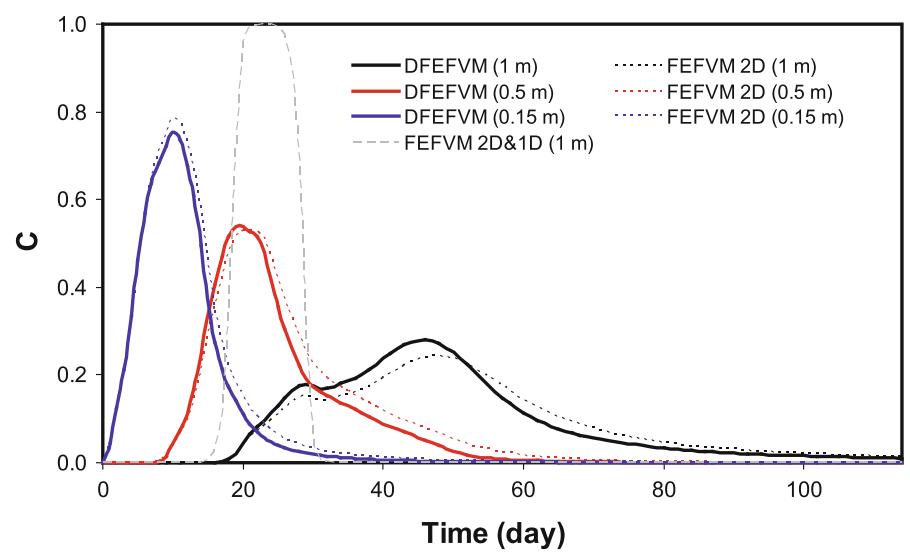

Fig. 20 Comparison of breakthrough curves calculated along at cross sections $0.15,0.5$, and $1 \mathrm{~m}$ away from injection boundary, i.e., top boundary (flow impediment model). The gray dashed line (FEFVM 1D \& 2D) presents the result of using lower dimensional elements for the flow impediments

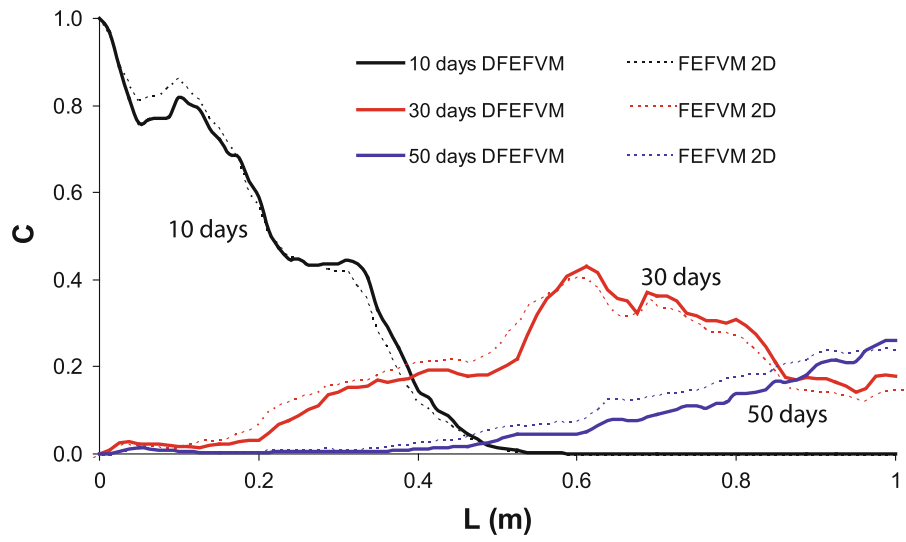

Fig. 21 Concentration profiles along the main flow direction at three different times (flow impediment model)

Fig. 22 Total mass calculated by the FEFVM 2D and DFEFVM (flow impediment model)

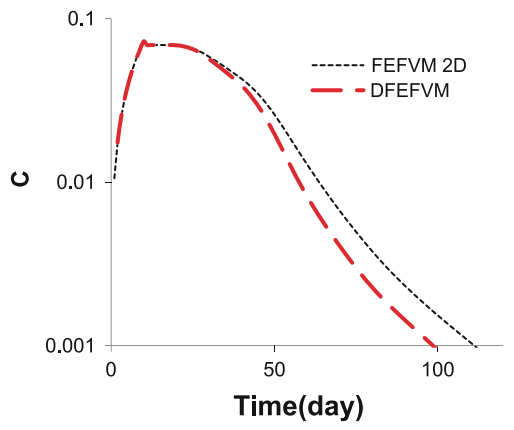

interfaces. Water breakthrough time and sweep efficiency are the main unknowns of interest to field-scale simulation. These are not predicted precisely by the FEFV methods for this model (Fig. 24). This is also revealed by Fig. 25 where total $S_{n}$ decreases faster using the 

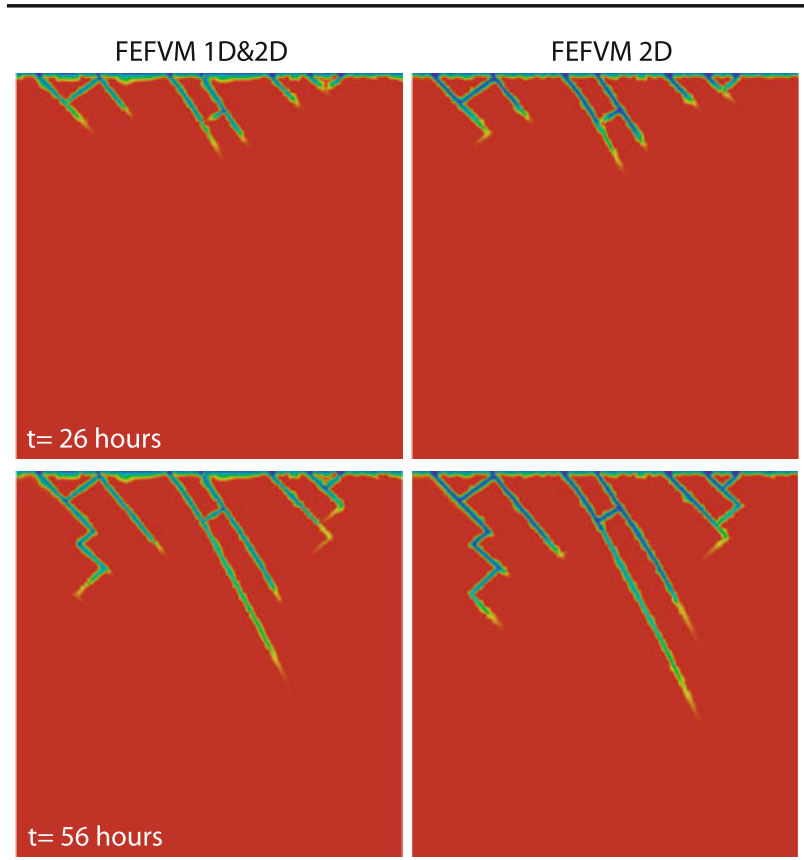

\section{DFEFVM 2D}
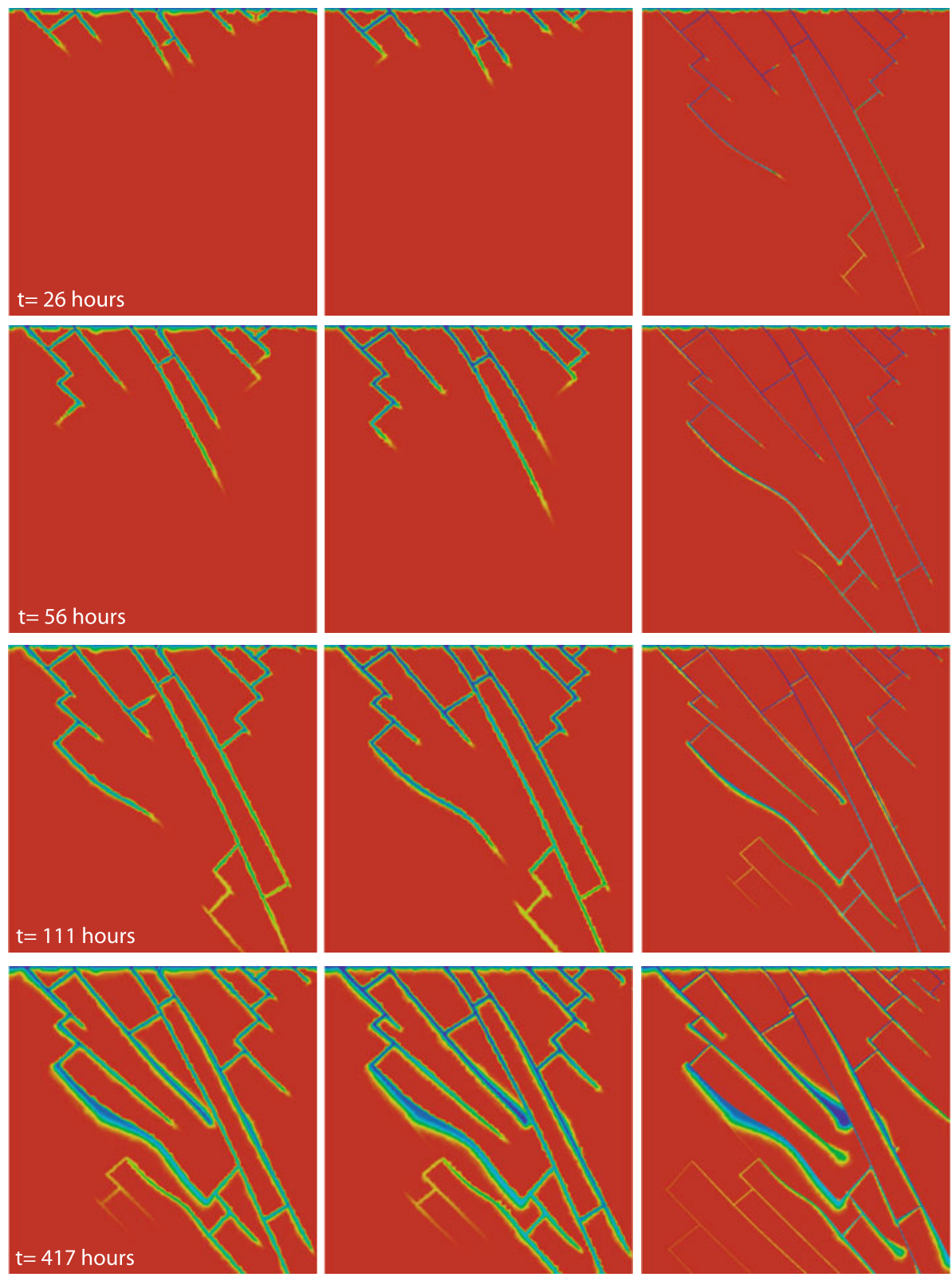

Oil Saturation

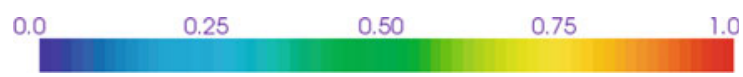

Fig. 23 Saturation fronts for Bristol channel model at four different times calculated by the three numerical schemes 


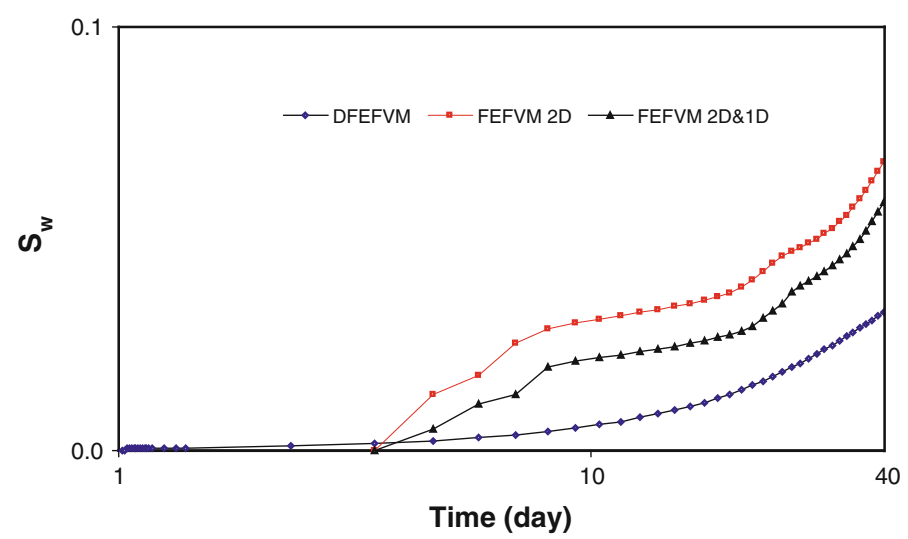

Fig. 24 Water saturation versus time at the outlet of the Bristol channel model

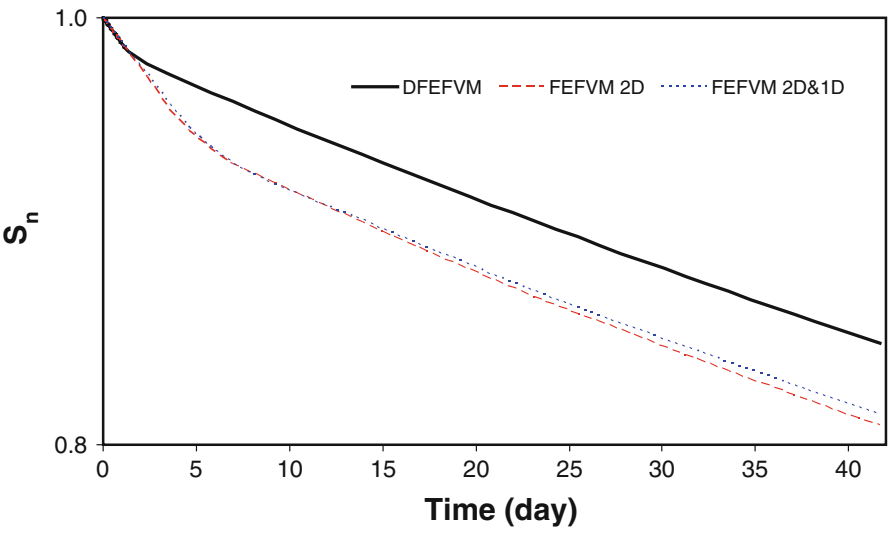

Fig. 25 Total oil saturation in Bristol channel model calculated by different numerical methods

FEFVM than for the DFEFVM, implying a more sweep when FEFVM is used. The total $S_{n}$ is calculated by $\sum_{j} \int_{v} \mathbf{M} \phi S_{n}^{t} d V / \sum_{j} \int_{v} \mathbf{M} \phi d V$.

\section{Discussion}

The single- or two-phase flow simulation using FEFVM 1D \& 2D needs a very fine mesh. Hoteit and Firoozabadi $(2008 a, b)$ argue that it is not practical to employ such a method on the large-scale. Using a very detailed mesh makes simulation of large-scale model computationally very costly. FEFVM representing fractures by lower dimensional line elements is used frequently for DFM simulations because this method resolves the computational problems posed by fracture elements with very large aspect ratios, see Juanes et al. (2002) and Paluszny et al. (2007). Apart from this advantage, however, this continuum method introduces errors into the simulation of single- and two-phase transport through fractured porous media due to the abrupt changes of the material properties across the fracture-matrix interfaces. This is not the case for the DFEFVM. In order to bring the advantages of the mixed dimensional method into the DFEFVM, it would need to be extended to discretizations with lower dimensional elements representing the fractures. This method would combine the ease of discretizing 
fractured media with the accuracy of the DFEFVM. Such a method is highly desirable for modeling dense fracture networks with a relatively coarse mesh.

The discussed DFEFVM is implemented explicitly in time and therefore suffers from the CFL constraint, the main drawback is that small finite volumes with large flow velocities cause the whole simulation to slow down. This can be improved by employing implicit time stepping (Matthäi et al. 2010). In many real fracture model simulations, fracture flow velocities and mesh refinement are 2-6 orders of magnitude greater than in the rock matrix. Already in the explicit form, DFEFVM facilitates using larger time steps as it does not require very fine elements for the fractures as opposed to the FEFVM which needs highly refined discretizations at fracture-matrix interfaces.

The DFEFVM provides an excellent framework for considering capillary effects at the interfaces. Benes et al. (2005), Mikyska (2005), Reichenberger et al. (2006), and Hoteit and Firoozabadi (2008a,b) show that saturation discontinuities caused by capillary effects can not be represented by the FEFVM unless a special treatment is employed. The tests described here show that DFEFVM can be employed to solve such a problem by adding capillary diffusion term in Eq. 6. This is subject to our ongoing research.

\section{Conclusions}

In this article, we have compared and contrasted three hybrid discretization schemes for flow in porous media: FEFVM, FEFVM on mixed dimensional elements, and DFEFVM. We have applied these three methods to single- and two-phase flow 2D models excluding gravitational and capillary terms. This detailed comparison highlights their differences. The results indicate that

- The FEFV continuum methods do not represent the real physics of single- or two-phase flow in fractured media when using a coarse mesh, due to smearing of the transport variable leading to inaccurate concentrations near the discontinuity.

- This also slows down the advance of saturation or concentration fronts in more permeable regions. Resulting transport speeds are not consistent with the permeability of the fractures. The opposite is true for low permeability regions.

- The DFEFVM resolves these issues and allows using a relatively coarse mesh to maintain accuracy.

- The DFEFVM predicts breakthrough times most accurately when compared to the FEFV continuum methods.

- Embedding discontinuities at the fracture-matrix interfaces, therefore, will lead to more accurate predictions of tracer transport and propagation speed of saturation fronts.

Acknowledgments We would like to thank the sponsors of the ITF project on Improved Simulation of Flow in Fractured and Faulted Reservoirs, and the Technology Strategy Board (TSB) for supporting this research. Chris C. Pain and Martin J. Blunt, receive our thanks for their feedback and comments.

Open Access This article is distributed under the terms of the Creative Commons Attribution Noncommercial License which permits any noncommercial use, distribution, and reproduction in any medium, provided the original author(s) and source are credited.

\section{References}

Bastian, P.: Numerical computation of multiphase flows in porous media. Habilitationsschrift, Universität Kiel (1999) 
Belayneh, M.: Paleostress orientation inferred from surface morphology of joints on the southern margin of the bristol channel basin, UK. In: Cosgrove, J.W., Engelder, T. (eds.) The Initiation, Propagation, and Arrest of Joints and Other Fractures, Special Publication 231, pp. 243-255. Geological Society Publishing House, Bath (2004)

Benes, M., Illangasekare, T., Mikyska, J.: On the numerical treatment of sharp texture transport in two-phase flow. In: Proceedings of the Czech-Japanese Seminar in Applied Mathematics, pp. 106-116, Oita (2005)

Brooks, R.H., Corey, A.T.: Hydraulic properties of porous media. Hydrology Papers, Colorado State University, 24 p. Colorado (1964)

Buckley, S., Leverett, M.: Mechanism of fluid displacement in sands. TAIME 146, 107-1116 (1942)

Chen, Z., Huan, G., Li, B.: An improved impes method for two-phase flow in porous media. Transp. Porous Media 54(3), 361-376 (2006)

Dietrich, P., Helmig, R., Sauter, H., Hötzl, H., Kongeter, J., Teutsch, G.: Flow and transport in fractured porous media. Springer, Berlin (2005)

Durlofsky, L.: A triangle based mixed finite element- finite volume technique for modeling two phase flow through porous media. J. Comput. Phys. 105(2), 252-266 (1998)

Eikemo, B., Lie, K.A., Dahle, H., Eigestad, G.: A discontinuous galerkin method for transport in fractured media using unstructured triangular grids. Adv. Water Res. 32(4), 493-506 (2009)

Geiger, S., Matthäi, S., Niessner, J., Helmig, R.: Black-oil simulations for three-component, three-phase flow in fractured porous media. SPE J. 6, 338-354 (2009)

Geiger, S., Cortis, A., Birkholzer, J.: Upscaling solute transport in naturally fractured porous media with the continuous time random walk method. Water Resour. Res. 46, W12530, 13p. (2010). doi:10.1029/ 2010WR009133

Gong, B., Karimi-Fard, M., Durlofsky, L.: Upscaling discrete fracture characterizations to dual-porosity, dual-permeability models for efficient simulation of flow with strong gravitational effects. SPE J. 13(1), 58-67 (2008)

Hoteit, H., Firoozabadi, A.: An efficient numerical model for incompressible two-phase flow in fractured media. Adv. Water Resour. 31(6), 891-905 (2008)

Hoteit, H., Firoozabadi, A.: Numerical modeling of two-phase flow in heterogeneous permeable media with different capillarity pressures. Adv. Water Resour. 31(1), 56-73 (2008)

Huber, R., Helmig, R.: Multi-phase flow in heterogeneous porous media: a classical finite element method versus an implicit pressure-explicit saturation-based mixed finite element-finite volume approach. Int. J. Numer. Methods Fluids 29(8), 899-920 (1999)

Huber, R., Helmig, R.: Node-centered finite volume discretizations for the numerical simulation of multiphase flow in heterogeneous porous media. Comput. Geosci. 4(2), 141-164 (2000)

Juanes, R., Samper, J., Molinero, J.: A general and efficient formulation of fractures and boundary conditions in the finite element method. Int. J. Numer. Methods Eng. 54(12), 1751-1774 (2002)

Karimi-Fard, M., Firoozabadi, A.: Numerical simulation of water injection in fractured media using the discrete-fracture model and the galerkin method. SPE J. 6(2), 117-126 (2003)

Kim, J., Deo, M.: Finite element, discrete-fracture model for multiphase flow in porous media. AIChE J. 46(6), 1120-1130 (2000)

Matthäi, S., Geiger, S., Roberts, S., Paluszny, A., Belayneh, M., Burri, A., Mezentsev, A., Lu, H., Coumou, D., Driesner, T., Heinrich, C.A.: Numerical simulation of multi-phase fluid flow in structurally complex reservoirs. In: Jolley, S.J., Barr, D., Walsh, J.J., Knipe, R.J. (eds.) Structurally Complex Reservoirs, Special Publication 292, pp. 405-429, Geological Society, London (2007)

Matthäi, S., Nick, H.: Upscaling two phase flow in naturally fractured reservoirs. AAPG Bull. 93(11), 1621-1632 (2009)

Matthäi, S., Nick, H., Pain, C., Neuweiler, I.: Simulation of solute transport through fractured rock: a higher-order accurate finite-element finite-volume method permitting large time steps. Transp. Porous Media 83(2), 289-318 (2010)

Mikyska, J.: Numerical model for simulation of behaviour of non-aqueous phase liquids in heterogeneous porous media containing sharp texture transitions. Ph.D. thesis, Czech technical university in Prague (2005)

Monteagudo, J.E.P., Firoozabadi, A.: Control-volume method for numerical simulation of two-phase immiscible flow in two- and three- dimensional discrete-fractured media. Water Resour. Res. 40, W07405, 20 p. (2004). doi:10.1029/2003WR002996

Nick, H., Matthäi, S.: A hybrid finite-element finite-volume method with embedded discontinuities for solute transport in heterogeneous media. Vadose Zone J. 10(1), 299-312 (2011)

Niessner, J., Helmig, R., Jakobs, H., Roberts, J.: Interface condition and linearization schemes in the Newton iterations for two-phase flow in heterogeneous porous media. Adv. Water Resour. 28(7), 671-687 (2005) 
Paluszny, A., Zimmerman, R.: Numerical simulation of multiple 3D fracture propagation using arbitrary meshes. Comput. Methods Appl. Mech. Eng. 200(9-12), 953-966 (2011)

Paluszny, A., Matthäi, S., Hohmeyer, M.: Hybrid finite element finite volume discretization of complex geologic structures and a new simulation workflow demonstrated on fractured rocks. Geofluids 7 , 186-208 (2007)

Rangel-German, E., Kovscek, A.: Matrix-fracture transfer functions for partially and completely immersed fractures. Twenty-Eighth Workshop on Geothermal Reservoir Engineering, pp. 1-9, Stanford University, Stanford, January (2003)

Reichenberger, V., Jakobs, H., Bastian, P., Helmig, R.: A mixed-dimensional finite volume method for twophase flow in fractured porous media. Adv. Water Resour. 29(7), 1020-1036 (2006)

$\mathrm{Su}, \mathrm{G}$. , Nimmo, J.: Effect of isolated fractures on accelerated flow in unsaturated porous rock. Water Resour. Res. 39(12), 1326-1337 (2003)

Unsal, E., Matthèi, S., Blunt, M.: Simulation of multiphase flow in fractured reservoirs using a fracture-only model with transfer functions. Comput. Geosci. 14(4), 527-538 (2010)

van Duijn, C., Molenaar, J., de Neef, M.: The effect of capillary forces on immiscible two-phase flow in heterogeneous porous media. Transp. Porous Media 21(71), 193-201 (1995)

van Genuchten, M.: A closed-form equation for predicting the hydraulic conductivity of unsaturated soils. Soil Sci. Soc. Am. J. 44, 892-898 (1980)

Zaretskiy, Y., Geiger, S., Sorbie, K., Foerster, M.: Efficient flow and transport simulations in reconstructed 3D pore geometries. Adv. Water Resour. 33(12), 1508-1516 (2010) 